\section{AB0243 CONSIDERING THE COMBINATION OF TWO BIOLOGICS IN CASE OF FAILURE OF THE MONOTHERAPY IN JUVENILE ONSET RHEUMATOID ARTHRITIS}

A. Haddouche ${ }^{1}$, K. Ait Bellabas ${ }^{1}$, W. F. Hamrani ${ }^{1}$, S. Sahraoui ${ }^{1}$, R. Fatma ${ }^{1}$, F. Rahal ${ }^{2}$, S. Slimani ${ }^{3}$, F. Hanni'. ${ }^{1}$ EHS Ben Aknoun, Rheumatology, Algiers, Algeria; ${ }^{2} \mathrm{CHU}$ Beni Messous, Rheumatology, Algiers, Algeria; ${ }^{3}$ Private Practice Office, Rheumatology, Batna, Algeria

Background: The management of rheumatoid arthritis refractory to conventional synthetic disease-modifying antirheumatic drugs (csDMARDs) is currently well codified and includes different types of biologics and even targeted sDMARDs. A rotation of biologic therapies is recommended in order to better control the disease.

Methods: We report the case of a 20-year-old patient followed in our hospital for the management of a deforming and erosive seropositive rheumatoid arthritis $(\mathrm{FR}+, \mathrm{ACPA}+)$ with a juvenile onset at the age of 8 years. The diagnosis of an immunopositive polyarticular form of JIA was retained in 2010 (9 years old); the patient was treated with methotrexate (MTX) at a dose of $10 \mathrm{mg}$ per week and methylprednisolone at doses varying between 4 and $10 \mathrm{mg}$ per day. Following the failure of MTX, etanercept was introduced for 6 months without success, followed by tocilizumab in 2012 at a dose of $8 \mathrm{mg} / \mathrm{kg} / \mathrm{month}$ for a year, without good response. In 2014, a course of rituximab (RTX) at a dose of 2 shots of $500 \mathrm{mg}$ 2 weeks apart was prescribed followed 9 months later by etanercept at a dose of $50 \mathrm{mg}$ a week for 3 years then by adalimumab $(40 \mathrm{mg} /$ week) because of the multiple treatment failures.

In 2018, the repetition of RTX at a dose of $1 \mathrm{~g}$, renewed 15 days later, improved the patient for only 3 months. Then, a combination of two biologics, namely RTX ( $2 \times 1 \mathrm{~g}, 15$ days apart) and adalimumab 1 month later ( $40 \mathrm{mg} /$ week) was received by the patient with a good response at 3 months. The latter was maintained for 7 months even after stopping the adalimumab following confinement for COVID-19. In September 2020, flares occurred and the adalimumab (ADA) has been delivered but without success during 3 months, stopped later for a benign form of COVID-19 (15 months after RTX). In January 2021, the association RTX + ADA was given again and we hope that it will be as effective as the first prescription

Results: The clinical and biological severity of our patient's rheumatoid arthritis led us to give a combination of two biological treatments. Indeed, we do not have other therapeutic classes to deliver to her, that encouraged us to rotate between all the available biological therapies in our country. The combination of a CD20 inhibitor (RTX) with a TNF blocker (ADA) was safe and made possible, for the first time, the achievement of clinical and biological remission during 7 months, even after stopping the TNF blocker. Greenwald et al. reported the safety of the combination of RTX + TNF inhibitors in a randomized clinical trial in 51 patients. Its efficacy, a secondary goal of the study, was suggested at 24 weeks by the percentage of ACR 20 and ACR 50 responses that was greater than in the RTX placebo group

Conclusion: The combination of RTX with a TNF blocker can be a real alternative therapy in rheumatoid arthritis with failure to a biological monotherapy.

Disclosure of Interests: None declared

DOI: 10.1136/annrheumdis-2021-eular.4058

\section{AB0244 INFLUENCE OF BIOLOGICAL DRUGS ON HEALTH- RELATED QUALITY OF LIFE IN PATIENTS WITH RHEUMATOID ARTHRITIS}

S. Jriri' ${ }^{1}$, S. Boussaid ${ }^{1}$, S. Rekik ${ }^{1}$, M. Abbes ${ }^{1}$, M. Ben Majdouba ${ }^{1}$, S. Jemmali ${ }^{1}$ H. Sahli ${ }^{1}$, M. Elleuch ${ }^{1} .{ }^{1}$ RABTA, Rhuematology, Tunis, Tunisia

Background: The level of the Health-related quality of life (HRQL) in patients with rheumatoid arthritis (RA) is often negleglected in their medical care. While theese patients are suffering from a precarious quality of life, resulting from pain impaired physical function and fatigue. The use of biological agents for treating this disease is then a challenge, leading to the possibility of reducing the consequences of the disease.

Objectives: The main purpose of this study was to compare the level of HRQL in patients with rheumatoid arthritis (RA) during therapy applying disease-modifying antirheumatic drugs (DMARDs) with conventional synthetics (cSDMARDs) or with csDMARDs in combination with biological drugs (bDMARDs).

Methods: The study involved 120 patients with RA, divided into two groups: group I -treated using csDMARDs (combination therapy: methotrexate and salazopyrine), group II - using csDMARDs in association with bDMARDs which included TNF inhibitors (etanercept and adalimumab). All the studied patients were surveyed with the use of the following questionnaires: the short-form health survey (SF-36) for HRQL that assesses eight domains: functional capacity (ten items), physical aspects (four items), pain (two items), general health (five items), vitality (four items), social aspects (two items), emotional issues (three items) and mental health (five items), in addiction to one item to compare current health status and that of the previous year, The AIMS2-SF, and Health Assessment Questionnaire (HAQ). The questionnaires were filled out at the consultation afte patient's consent.The 28-Joint Disease Activity Score (DAS-28) was calculated. Results: Group I consisted of 72 persons including 55 women and 17 men with a mean age of 58.4 years. Group II contaned 48 patients where females predominated (sex ratio: 0.3 ), the mean age was 52.4 years. The majority of patients $(53.3 \%)$ had been diagnosed with RA for more than five years. Most of the SF-36 domains showed significant improvement in the second group $(p<0.01)$, high lighting the social aspects, pain, physical functioning, emotional issues, vitalty and physical aspects. The mean score of HAQ II decreased from 1.97 up to 1.23 with biological therapy $(p<0.01)$. The highest AIMS scores were comparatively in the two groups (I vs II): in social activity $(6.49 \pm 1.93$ vs $6.23 \pm 1.56)$, pain $(4.70 \pm 2.04$ vs $4.01 \pm 2)$, depression $(4.70 \pm 2.23$ vs $4.66 \pm 2.03)$, and physical activ ity $(4.03 \pm 2.10$ vs $4.01 \pm 2.08)$. The DAS-28 value, the number of swollen joints, and the duration of morning stiffness were significantly smaller among patients from group II $(P=0.04)$. After logistical regression, treatement with biotherapy was isolated as a fundamental independent factor influencing the mentel component of SF-36 scale with an OR of 1.59 .

Conclusion: we conclude that the use of biologic therapy in patients with RA proved to be an important pharmacological strategy for improving HRQL and functional capacity as assessed by the HAQ II and SF-36 instruments. The intensity of the activity of RA as well as experiencing pain and the duration of morning stiffness were smaller among patients applying csDMARDs plus bDMARDs compared with patients treated only with csDMARDs.

\section{REFERENCES:}

[1] Blair, H. A., \& Deeks, E. D. (2016). Infliximab Biosimilar (CT-P13; Infliximab-dyyb): A Review in Autoimmune Inflammatory Diseases. BioDrugs, 30(5), 469-480.

[2] Araújo, F., Gonçalves, J., \& Fonseca, J. E. (2016). Biosimilar DMARDs: What Does the Future Hold? Drugs, 76(6), 629-637.

Disclosure of Interests: None declared

DOI: 10.1136/annrheumdis-2021-eular.4223

\section{$\mathrm{AB} 0245$}

ANALYSIS OF THE CLINICAL AND ANTIDESTRUCTIVE EFFECTS OF RITUXIMAB DEPENDING ON GENDER IN PATIENTS WITH RHEUMATOID ARTHRITIS

A. Kudryavtseva ${ }^{1}$, G. Lukina ${ }^{1}$, E. Aronova ${ }^{1}$, G. Gridneva ${ }^{1}$, S. Glukhova ${ }^{1}$

A. Smirnov ${ }^{1}$. $V$.A. Nasonova Research Institute of Rheumatology, Laboratory

for the Study of Comorbid Infections and Monitoring the Safety of Therapy, Moscow, Russian Federation

Background: Rheumatoid arthritis is a chronic autoimmune disease characterized by inflammation of the synovial tissue and destruction of the underlying cartilage and bones. It was found that RA more often affects women than men, with a sex ratio of $3: 1$. And the question of the influence of gender on the outcomes and course of RA remains controversial, there is no consensus on whether RA is worse in women or men. Recent reports indicate that women are less likely to achieve remission than men. Women suffer from RA at an earlier age and have higher markers of disease activity such as DAS28 and HAQ. Rituximab is a chimeric monoclonal antibody that targets the CD20 molecule expressed on the surface of B cells, it has been successfully and widely used for the treatment of rheumatoid arthritis, so it is of interest to assess whether gender influences the therapeutic and radiological effects of RTX.

Objectives: The aim of this study was to analyze the impact of gender on the response to rituximab (RTX) in patients with $\mathrm{RA}$.

Methods: Initially, 221 women(w), $27 \operatorname{men}(m)$, were examined to assess the clinical and X-ray effect $(88 \mathrm{w} / 6 \mathrm{~m})$, who received RTX treatment (1000 mgx2 or $500 \mathrm{mgx} 2$ ). Both groups were comparable in terms of the main clinical and laboratory characteristics (age, duration of the disease, the number of preceding DMARDs, in both groups most patients were RF + and ACCP + a high degree of activity according to DAS 8 - men - 5.6 [4.6-6.7], women 6.04 [5.2-6.63] Initially, the degree of radiological changes in men is slightly higher than in women ( $p>0.05)$. Clinical effect was scored by EULAR criteria radiographic progression was assessed using Sharp/van der Heijde modified scoring method.

Results: When assessing the clinical effect after 48 weeks in men, a significantly better effect of RTX treatment was noted in comparison with women ( $\triangle$ DAS28, a significantly better effect was noted in men $-\Delta$ DAS28 $=3.75[2.8-4.14]$, and $\Delta$ DAS28 $=1.3[0.37-2.72]$ in women, $(p=0.04)$. Analyzing the X-ray effect after 48 weeks of RTM treatment: the absence of progression in terms of the total score in $83.33 \%$ of men and $60.98 \%$ of women; there was no progression in narrowing of the joint space in $83.33 \%$ of men and $65.85 \%$ of women, noteworthy that the account of erosion practically reaches statistical significance - inhibition of destruction in $100 \%$ of men and $74.31 \%$ of women $(p=0.06)$

Conclusion: Thus, having analyzed the clinical and antidestructive effects of RTM therapy depending on gender, we can conclude that the effect is significantly 
higher in the male group. Also, there is a tendency towards more effective inhibition of radiographic progression in men.

Disclosure of Interests: None declared

DOI: 10.1136/annrheumdis-2021-eular.4262

\section{AB0246 COMORBIDITIES APPEARING UNDER BIOLOGIC THERAPY: PREVALENCE AND ASSOCIATED FACTORS}

T. El Joumani ${ }^{1}$, T. Latifa ${ }^{1}$, I. Hmamouchi ${ }^{2,3}$, S. Ahid ${ }^{4}$, R. Abouqal ${ }^{5}$, L. Achemlal ${ }^{6}$, I. El Bouchti ${ }^{7}$, A. El Maghraoui ${ }^{8}$, I. Ghozlani ${ }^{9}$, H. Hassikou $^{10}$ T. Harzy ${ }^{11}$, I. Linda ${ }^{12}$, O. Mkinsi ${ }^{13}$, R. Niamane ${ }^{14}$, R. Bahiri ${ }^{15}$, H. Rkain ${ }^{1}$, F. Allali'. ' El Ayachi Hospital, Faculty of Medicine and Pharmacy of Rabat, Mohammed V University, Rabat, Morocco, Department of Rheumatology B, Rabat, Morocco; ${ }^{2}$ Faculty of Medicine and Pharmacy, Mohammed V University, Rabat, Morocco, Laboratory of Biostatistical, Clinical and Epidemiological Research, Rabat, Morocco; ${ }^{3}$ Provincial Hospital of Temara, Morocco, Department of Rheumatology, Rabat, Morocco; ${ }^{4}$ Mohammed $\checkmark$ University, Rabat, Morocco, Research Team of Pharmacoeconomics \& Pharmacoepidemiology, Rabat, Morocco; ${ }^{5}$ Faculty of Medicine and Pharmacy of Rabat, Mohammed V University, Rabat, Morocco, Laboratory of Biostatistical, Clinical and Epidemiological Research, Rabat, Morocco; ${ }^{6}$ Military Hospital Mohammed V, Ibn Sina University Hospital, Rabat, Morocco, Department of Rheumatology, Rabat, Morocco; ${ }^{7}$ Arrazi University Hospital, Marrakech, Morocco, Department of Rheumatology, Rabat, Morocco; ${ }^{8}$ Private Medical Office, Rabat, Morocco, Rheumatology, Rabat, Morocco; ${ }^{9}$ University Hospital of Agadir, Morocco, Department of Rheumatology, Agadir, Morocco; ${ }^{10}$ Military Hospital Moulay Ismail, Hassan II University Hospital, Meknès, Morocco, Department of Rheumatology, Meknes, Morocco: ${ }^{11}$ Hassan II University Hospital, Fès, Morocco, Department of Rheumatology, fes, Morocco; ${ }^{12}$ Mohammed VI University Hospital, Department of Rheumatology, Oujda, Morocco: ${ }^{13} \mathrm{Ibn}$ Rochd University Hospital, Department of Rheumatology, casablanca, Morocco: ${ }^{14}$ Military Hospital Avicenne, Mohammed VI University Hospital, Department of Rheumatology, marrakech, Morocco; ${ }^{15}$ El Ayachi Hospital, Ibn Sina University Hospital, Department of Rheumatology A, Salé, Morocco

Objectives: The aims of our study are to determine the new comorbidities appearing under biologic therapy, their prevalence, and the factors implicated in their appearance.

Methods: It's a multicentric historical-prospective cohort including 10 rheumatology departments of Moroccan University Hospitals. The data were collected from the national register of patients under biologic therapy supervised by the Moroccan Society of Rheumatology. An electronic follow-up questionnaire is completed every 6 months by the investigator.

Results: The study included 418 patients: 224 with Rheumatoid Arthritis (RA) who represented $53.6 \%$ and 194 with spondyloarthropathy (SP) who represented $46.4 \%$.

The prevalence rate of comorbidities appearing after one year of treatment with biologic therapy was $15.7 \%$ in RA and $8.4 \%$ in SP. The rate of cardiovascular diseases was $12.9 \%$ (arterial hypertension, myocardial infarction and/or ischemic stroke), $6.4 \%$ was the same value of hypercholesterolemia and depression, diabetes $3.3 \%$, ulcer $3.2 \%$, and osteoporosis $9.7 \%$. The sedentary rate was $54.8 \%$ and smoking was about $3.3 \%$.

No patient had developed hypertriglyceridemia or chronic obstructive pulmonary disease.

In the group of patients with RA, the average age of the patients who had developed a new comorbidity was $51,8 \pm 11,3$ years, women represented $87.5 \%$, the average of Body Mass Index (BMI) was 27,6 $\pm 5,9$ and the average duration of the disease was $14,1 \pm 9,2$ years. The disease activity score (DAS28) had an average of $3,15 \pm 1,47$.

$59.6 \%$ of patients used Rituximab, 23.8\% Tociluzimab, 8.1\% Etanercept, $5.8 \%$ Adalimumab, $0.18 \%$ Infliximab, and $0.9 \%$ Golimumab

In the group of patients with SP, the average age of the patients who had developed a new comobidity was $40,2 \pm 13,7$ years, men represented $63.4 \%$, and the average of BMI was $24,3 \pm 4,94$

The activity of the desease, had an average Bath Ankylosing Spondylitis Disease Activity Index (BASDAl) of 2,62 $\pm 1,79$ and ankylosing Spondylitis Disease Activity Score (ASDAS) of $1,93 \pm 1,09$.

Regarding the type of biologic therapy, $33.2 \%$ of patients used Etanercept, $30.1 \%$ Adalimumab, $24.9 \%$ Infliximab, 9.8\% Golimumab, 1.6\% Secukinumab, and $0.5 \%$ Tociluzimab.

Conclusion: Our study showed a high prevalence of cardiovascular disease in patients under biologic therapy. This can be explained by the sedentary lifestyle secondary to rheumatic disease.

Disclosure of Interests: None declared

DOI: 10.1136/annrheumdis-2021-eular.4280

\section{Rheumatoid arthritis - non biologic treatment and small molecules}

\section{$\mathrm{AB} 0247$ \\ ANALYSIS OF THE IMPACT OF TOFACITINIB TREATMENT ON WEIGHT IN PATIENTS WITH RHEUMATOID ARTHRITIS}

J. Wollenhaupt ${ }^{1}$, J. Morel ${ }^{2}$, C. Daien ${ }^{2}$, A. Ruyssen-Witrand ${ }^{3}$, C. Lukas ${ }^{2}$, C. Richez ${ }^{4}$, A. Shapiro ${ }^{5}$, D. Chapman ${ }^{6}$, M. Cros $^{7}$, J. L. Rivas ${ }^{8}$, G. Citera ${ }^{9}$ ${ }^{1}$ Struenseehaus Centre for Rheumatology and Clinical Immunology, Hamburg, Germany; ${ }^{2} \mathrm{CHU}$ Montpellier and University of Montpellier, Department of Rheumatology, Montpellier, France; ${ }^{3}$ Hopital Purpan, CHU Toulouse, Department of Rheumatology, Toulouse, France; ${ }^{4}$ Groupe Hospitalier Pellegrin-CHU de Bordeaux, Department of Rheumatology, Bordeaux, France; ${ }^{5}$ Pfizer Inc, Inflammation and Immunology, Peapack, NJ, United States of America; ${ }^{6}$ Pfizer Inc, Inflammation and Immunology, New York, NY, United States of America; ${ }^{7}$ Pfizer Inc, Inflammation and Immunology, Paris, France; ${ }^{8}$ Pfizer SLU, Inflammation and Immunology, Madrid, Spain; ${ }^{9}$ Instituto de Rehabilitación Psicofísica, Section of Rheumatology, Buenos Aires, Argentina

Background: A prior post hoc analysis of tofacitinib clinical trial data reported improvements in rheumatoid arthritis (RA) outcomes with tofacitinib vs placebo (PBO) through Month (M)6, regardless of baseline (BL) body mass index (BMI). Objectives: To assess change from BL $(\Delta)$ in BMI, and disease activity by $\mathrm{BL} B M I$ status, in patients (pts) with moderate/severe RA receiving tofacitinib through M12.

Methods: This post hoc analysis included data pooled from Phase 3 and $3 b / 4$ studies of pts who were methotrexate-naïve (NCT01039688) or inadequate responders to conventional synthetic (cs) or biologic DMARDs (NCT00960440; NCT00847613; NCT00814307; NCT00856544; NCT00853385; NCT02187055 NCT02831855). Pts received $\geq 1$ dose of tofacitinib 5 or $10 \mathrm{mg}$ twice daily (BID) or $11 \mathrm{mg}$ once daily (QD), \pm csDMARDs, or PBO. Least squares (LS) mean $\triangle \mathrm{BM}$ (linear mixed model repeated measures; observed cases) was summarised for all treatment groups at M3/6/12 (M3/6 only for tofacitinib $11 \mathrm{mg} Q D$ and PBO). Other assessments at $\mathrm{M} 3 / 6 / 12$ included $\Delta \mathrm{BMI} \pm \mathrm{BL}$ concomitant glucocorticoids (GCs) or antidepressants (descriptive statistics), LS mean $\triangle \mathrm{DAS} 28-4$ (ESR) stratified by BL BMI status $(<25, \geq 25-<30, \geq 30)$, and correlations between LS mean $\triangle \mathrm{BMI}$ and $\triangle \mathrm{DAS} 28-4(\mathrm{ESR})$.

Results: In total, 2349, 1611, 694 and 681 pts received tofacitinib $5 \mathrm{mg}$ BID, $10 \mathrm{mg}$ BID, $11 \mathrm{mg}$ QD or PBO, respectively. Demographics/baseline characteristics were generally similar across treatments, except for some numerical differences in the tofacitinib $11 \mathrm{mg}$ QD group, eg fewer female pts, more White pts and fewer pts receiving concomitant GCs, compared with other treatment groups. At M3/6, LS mean BMI significantly increased from BL with all tofacitinib doses vs PBO (all $\mathrm{p}<0.05$ ); LS mean $\triangle \mathrm{BMI}$ was greatest with $10 \mathrm{mg}$ $\mathrm{BID}$ and lowest with $11 \mathrm{mg}$ QD (Figure 1a). LS mean $\triangle \mathrm{BMI}$ was greater in pts receiving tofacitinib as monotherapy vs combination therapy at $\mathrm{M} 3 / 6 / 12$ (Figure $1 \mathrm{~b}) . \triangle \mathrm{BMI}$ was generally similar in pts receiving treatment \pm concomitan GCs or antidepressants (data not shown). Improvements in DAS28-4(ESR) were observed in each $B L B M I$ status group at $M 3 / 6 / 12$ and were greatest with all tofacitinib doses vs PBO. LS mean $\triangle \mathrm{DAS} 28-4$ (ESR) was generally numerically highest for pts with $\mathrm{BMI}<25$ and numerically lowest for pts with BMI $>30$, for all tofacitinib doses. LS mean $\triangle \mathrm{DAS} 28-4(\mathrm{ESR})$ was generally greatest with tofacitinib $10 \mathrm{mg}$ BID and $11 \mathrm{mg}$ QD vs $5 \mathrm{mg}$ BID across BL BM status groups (Figure 1C). Across treatments, model-adjusted associations between LS mean $\triangle \mathrm{DAS} 28-4(\mathrm{ESR})$ and $\triangle \mathrm{BMI}$ were weak (correlation coefficients all $<0.3$; Table 1 ).

Table 1. Correlations between LS mean $\triangle \mathrm{DAS} 28-4(\mathrm{ESR})$ and $\triangle \mathrm{BMI}$ through M12

\begin{tabular}{lcccccccc}
\hline & \multicolumn{2}{c}{$\begin{array}{c}\text { Tofacitinib } \\
\mathbf{5} \mathbf{~ m g ~ B I D}\end{array}$} & \multicolumn{2}{c}{$\begin{array}{c}\text { Tofacitinib } \\
\mathbf{1 0} \mathbf{m g} \text { BID }\end{array}$} & \multicolumn{2}{c}{$\begin{array}{c}\text { Tofacitinib } \\
\mathbf{1 1} \mathbf{~ m g ~ Q D ~}\end{array}$} & PBO \\
\cline { 2 - 9 } & $\mathbf{N}$ & $\begin{array}{c}\text { Correlation } \\
\text { coefficient }\end{array}$ & $\mathbf{N}$ & $\begin{array}{c}\text { Correlation } \\
\text { coefficient }\end{array}$ & $\mathbf{N}$ & $\begin{array}{c}\text { Correlation } \\
\text { coefficient }\end{array}$ & $\mathbf{N}$ & $\begin{array}{c}\text { Correlation } \\
\text { coefficient }\end{array}$ \\
\hline M3 & 2021 & 0.1169 & 1348 & 0.1240 & 641 & 0.0907 & 554 & 0.0783 \\
M6 & 1918 & 0.1305 & 1270 & 0.1397 & 611 & 0.0438 & 150 & 0.1556 \\
M12 & 1455 & 0.1213 & 874 & 0.1826 & - & - & - & - \\
\hline
\end{tabular}

Slopes for associations between LS mean $\triangle \mathrm{BMI}$ and $\triangle \mathrm{DAS} 28-4(\mathrm{ESR})$ were significantly differ ent from 0 at $M 3 / 6 / 12$ with tofacitinib 5 and $10 \mathrm{mg} \mathrm{BID} \mathrm{(all} p<0.05$ ). Correlations were analysed by a general linear model method, which included $\mathrm{BL}$ age, gender, race and RA duration. For pts receiving tofacitinib $11 \mathrm{mg}$ QD in ORAL Shift (NCT02831855), only data to M6 were included. Pts who advanced from PBO to tofacitinib were not analysed post-advancementDAS28-4(ESR), Disease Activity Score in 28 joints, erythrocyte sedimentation rate; N, number of pts analysed 